\title{
Erratum: Many-body formalism for thermally excited wave packets: A way to connect the quantum regime to the classical regime [Phys. Rev. A 95, 062124 (2017)]
}

Aurélia Chenu and Monique Combescot

(Received 11 October 2017; published 30 October 2017)

DOI: 10.1103/PhysRevA.96.049907

The aim of this erratum is to give proper credit to previous research that inspired our work.

The splitting of the thermal energy used in Eq. (34) was first introduced by Hornberger and Sipe [1].

We thank John Sipe and Agata Brańczyk for pointing out this reference, and are grateful for the many helpful discussions.

[1] K. Hornberger and J. E. Sipe, Phys. Rev. A 68, 012105 (2003). 